\title{
TLR Agonist BDB001
}

National Cancer Institute

\section{Source}

National Cancer Institute. TLR Agonist BDB001. NCI Thesaurus. Code C156395.

A toll-like receptor (TLR) agonist with potential immunostimulating and antineoplastic activities. Upon administration, TLR agonist BDB001 activates one or more not yet disclosed TLRs, which may result in macrophage and plasmacytoid dendritic cell (pDC) stimulation; secretion of interferon alpha (IFNa); production of proinflammatory cytokines; upregulation of co-stimulatory molecules; enhanced T- and B-cell stimulatory responses; T-cell proliferation; and a T-helper 1 (Th1) immune response. TLRs are transmembrane receptors that recognize structurally conserved microbial molecules such as bacterial cell-surface lipopolysaccharides (LPS), lipoproteins, lipopeptides, lipoarabinomannan and flagellin, among others; immune responses stimulated by TLR activation may result in immune-mediated tumor cell killing. 